Bifurcation and forced symmetry breaking in Hamiltonian systems

Grabsi, Fethi and Montaldi, James and Ortega, Juan-Pablo

2004

MIMS EPrint: 2005.10

Manchester Institute for Mathematical Sciences

School of Mathematics

The University of Manchester

\footnotetext{
Reports available from: http://eprints.maths.manchester.ac.uk/

And by contacting: The MIMS Secretary

School of Mathematics

The University of Manchester

Manchester, M13 9PL, UK
} 


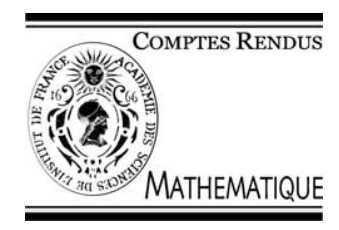

Differential Geometry/Dynamical Systems

\title{
Bifurcation and forced symmetry breaking in Hamiltonian systems
}

\author{
Féthi Grabsi ${ }^{a}$, James Montaldi ${ }^{\mathrm{b}}$, Juan-Pablo Ortega $^{\mathrm{c}}$ \\ a Institut non linéaire de Nice, UMR 129 CNRS-UNSA, 1361 route des Lucioles, 06560 Valbonne, France \\ ${ }^{\mathrm{b}}$ Department of Mathematics. UMIST, PO Box 88, Manchester M60 1QD, United Kingdom \\ ${ }^{\mathrm{c}}$ Centre national de la recherche scientifique, département de mathématiques de Besançon, Université de Franche-Comté, \\ 16, route de Gray, 25030 Besançon cedex, France \\ Received 7 January 2004; accepted 13 January 2004 \\ Presented by Charles-Michel Marle
}

\begin{abstract}
We consider the phenomenon of forced symmetry breaking in a symmetric Hamiltonian system on a symplectic manifold. In particular we study the persistence of an initial relative equilibrium subjected to this forced symmetry breaking. We see that, under certain nondegeneracy conditions, an estimate can be made on the number of bifurcating relative equilibria. To cite this article: F. Grabsi et al., C. R. Acad. Sci. Paris, Ser. I 338 (2004).
\end{abstract}

(c) 2004 Académie des sciences. Published by Elsevier SAS. All rights reserved.

\section{Résumé}

Bifurcation et brisure forcée de symétrie dans les systèmes hamiltoniens. Nous considérons le phénomène de brisure forcée de symétrie dans un système hamiltonien symétrique défini sur une variété symplectique. Plus précisement, nous étudions la persistance d'un équilibre relatif soumis à une brisure de symétrie. Nous verrons que, sous certaines hypothèses de nondégénéréscence, on peut donner une estimation du nombre d'équilibres relatifs persistants après la brisure. Pour citer cet article : F. Grabsi et al., C. R. Acad. Sci. Paris, Ser. I 338 (2004).

(C) 2004 Académie des sciences. Published by Elsevier SAS. All rights reserved.

\section{Version française abrégée}

Nous nous intéressons au phénomène de la brisure forcée de symétrie dans les systèmes hamiltoniens symétriques. On considère une action libre du tore $\mathbb{T}^{n}$ de dimension $n$ sur une variété symplectique $(\mathcal{M}, \omega)$ qui admet une application moment (nécessairement invariante) $\mathbf{J}_{\mathbb{T}^{n}}: \mathcal{M} \rightarrow\left(\mathfrak{t}^{n}\right)^{*} \simeq \mathbb{R}^{n}$. Soit $H_{0}$ une fonction hamiltonienne invariante par rapport à l'action de $\mathbb{T}^{n}$ et dont le champ de vecteurs $X_{H_{0}}$ associé présente, par hypothèse, un équilibre relatif (ER). Nous rappelons qu'un ER $m$ de $X_{H_{0}}$ est défini par la condition $X_{H_{0}}(m)=$ $\xi_{\mathcal{M}}(m)$, pour un élément $\xi$ dans l'algèbre de Lie de $\mathbb{T}^{n}$ dénommé la vitesse de l'ER.

E-mail addresses: fethi.grabsi@inln.cnrs.fr (F. Grabsi), j.montaldi@umist.ac.uk (J. Montaldi), Juan-Pablo.Ortega@math.univ-fcomte.fr (J.-P. Ortega). 
Soit $\mathbb{T}^{r} \subset \mathbb{T}^{n}$ un sous-tore de $\mathbb{T}^{n}$ et $H_{\varepsilon}$ une famille de perturbations $\mathbb{T}^{r}$-invariantes de l'hamiltonien $H_{0}$, paramétrée d'une manière lisse par $\varepsilon \in \mathbb{R}$. En principe, les ER du système $\left(\mathcal{M}, \omega, H_{0}, \mathbb{T}^{n}, \mathbf{J}_{\mathbb{T}^{n}}\right)$ ne seront plus en général des ER pour le système $\left(\mathcal{M}, \omega, H_{\varepsilon}, \mathbb{T}^{r}, \mathbf{J}_{\mathbb{T}^{r}}\right)$. Notre intérêt est de déterminer sous quelles conditions l'ER de départ $m$ continue à être un ER du système $\left(\mathcal{M}, \omega, H_{\varepsilon}, \mathbb{T}^{r}, \mathbf{J}_{\mathbb{T}^{r}}\right)$. Plus précisement, nous montrons que les ER qui sont non-dégénérés dans un certain sens et dont la vitesse appartient à l'algèbre de Lie du sous-tore $\mathbb{T}^{r}$ persistent. De plus, le théorème suivant nous donne une estimation de leur nombre :

Théorème 1. Soit $H_{\varepsilon}$ une famille de fonctions hamiltoniennes paramétrisée d'une manière lisse par $\varepsilon \in \mathbb{R}$ et définie sur la variété symplectique $(\mathcal{M}, \omega)$. Supposons que $H_{0}$ est invariante par rapport à une action libre et canonique du tore $\mathbb{T}^{n}$ et que le champ de vecteurs associé $X_{H_{0}}$ a un équilibre relatif $m$ avec vitesse $\xi \in \mathfrak{t}^{n}$ et moment $\mu:=\mathbf{J}_{\mathbb{T}^{n}}(m) \in\left(\mathfrak{t}^{n}\right)^{*} \simeq \mathbb{R}^{n} . \mathbf{J}_{\mathbb{T}^{n}}: \mathcal{M} \rightarrow\left(\mathfrak{t}^{n}\right)^{*}$ est une application moment associée à l'action de $\mathbb{T}^{n}$.

Soit $\mathbb{T}^{r} \subset \mathbb{T}^{n}$ un sous-tore dont l'action restreinte associée sur $\mathcal{M}$ laisse invariantes les foncions $H_{\varepsilon}$, pour tout $\varepsilon$. Notons par $i: \mathfrak{t}^{r} \hookrightarrow \mathfrak{t}^{n}$ l'inclusion de l'algèbre de Lie de $\mathbb{T}^{r}$ dans celle de $\mathbb{T}^{n}$ et par $i^{*}:\left(\mathfrak{t}^{n}\right)^{*} \rightarrow\left(\mathfrak{t}^{r}\right)^{*}$ son dual. Si $m$ est $i^{*} \mu$-non-dégénéré dans le sens de la Définition 2.1 et sa vitesse appartient à l'algèbre de Lie de $\mathbb{T}^{r}$ alors pour chaque valeur du paramètre $\varepsilon$ suffisamment proche de 0 , les équilibres relatifs de $X_{H_{\varepsilon}}$ sont en correspondance bijective avec les points critiques d'une fonction lisse $\bar{h}_{\varepsilon}: \mathbb{T}^{n-r} \rightarrow \mathbb{R}$. Par conséquent, il existe au moins $n-r+1$ équilibres relatifs de $X_{H_{\varepsilon}}$ avec moment $i^{*} \mu$ et vitesse proche de $\xi$. De plus, si les points critiques de $\bar{h}_{\varepsilon}$ sont tous non-dégénérés le nombre d'équilibres relatifs bifurqués est au moins $2^{n-r}$.

Pour établir ce théorème, nous utilisons la technique de réduction symplectique par rapport au tore $\mathbb{T}^{r}$ (espace de Marsden-Weinstein $\mathcal{M}_{\alpha}$ ) dans les coordonnées de Marle-Guillemin-Sternberg correspondantes à l'action de $\mathbb{T}^{n}$. Nous y caractérisons les équilibres relatifs bifurquant de $m$ après brisure forcée de symétrie comme points critiques de l'hamiltonien réduit $h_{\varepsilon}$ sur $\mathcal{M}_{\alpha}$. Moyennant une condition de non-dégénérescence sur l'équilibre relatif initial $m$, nous obtenons de $h_{\varepsilon}$ une fonction $\bar{h}_{\varepsilon}$ sur le tore $\mathbb{T}^{n-r}$ dont les points critiques sont en correspondance bijective avec les ER recherchés. Il s'en suit par la théorie des points critiques d'une fonction à valeurs réelles sur une variété compacte les estimations sur leur nombre (catégorie de Ljusternik-Schnirelmann, théorie de Morse).

\section{Introduction}

Forced symmetry breaking in dynamical systems is a phenomenon that takes place when we add to a symmetric system a perturbation with less symmetry. In this Note we study this phenomenon in the context of globally Hamiltonian dynamical systems, that is, symmetric Hamiltonian systems to which a momentum map can be associated. The particular problem of study is the "survival" or persistence of relative equilibria of the fully symmetric system after the symmetry breaking perturbation is added to it. Our motivation relies strongly upon the fact that this phenomenon is naturally present in many systems. For instance, consider a spherical pendulum whose bob of mass $m$ has been charged with a positive charge $q$ (see Fig 1). Suppose now that right below the point of suspension of the pendulum we place a charge identical to that of the pendulum (position (a) in the picture). If the repulsive electrostatic force is strong enough, the stable downright equilibrium of the spherical pendulum becomes unstable and a ring of equilibria appears. Suppose now that the circular symmetry of the system is broken by slightly sliding the charge to a side (position (b) in the picture). It can be seen that only two of the equilibria

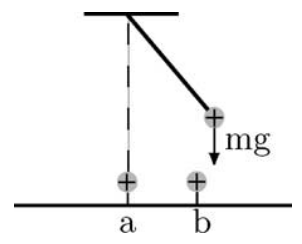

Fig. 1. 
in the ring survive. Our main goal in this paper is the formulation of a general theorem capable of predicting such behaviour. The kind of systems we are interested in can be mathematically described by considering a finitedimensional symplectic manifold $(\mathcal{M}, \omega)$ acted freely and canonically upon by the $n$-torus $\mathbb{T}^{n}$. We assume that this action has a momentum map $\mathbf{J}_{\mathbb{T}^{n}}: \mathcal{M} \rightarrow\left(\mathfrak{t}^{n}\right)^{*} \simeq \mathbb{R}^{n}$. Let $\mathbb{T}^{r} \subset \mathbb{T}^{n}$ be a subtorus, $H_{\varepsilon}$ a family of Hamiltonian functions on $\mathcal{M}$ parametrized by $\varepsilon \in \mathbb{R}$, and assume that $H_{0}$ is $\mathbb{T}^{n}$-invariant whereas $H_{\varepsilon}$ is only $\mathbb{T}^{r}$-invariant, for all $\varepsilon \in \mathbb{R}$. The problem that we discuss in this note is under what conditions a given relative equilibrium $m \in \mathcal{M}$ of $H_{0}$ with respect to its $\mathbb{T}^{n}$-symmetry persists to relative equilibria of the Hamiltonian vector fields associated to $H_{\varepsilon}$, for $\varepsilon$ sufficiently small, with respect to their $\mathbb{T}^{r}$-symmetry. We recall that a relative equilibrium (RE) of a $\mathbb{T}^{n}$-equivariant dynamical system $X$ on $\mathcal{M}$ is a point $m$ for which there exists an element $\xi$ in the Lie algebra $\mathfrak{t}^{n}$ of $\mathbb{T}^{n}$ (called the velocity of the RE) such that $X(m)=\xi_{\mathcal{M}}(m)$. The symbol $\xi_{\mathcal{M}}(m):=\left.\frac{\mathrm{d}}{\mathrm{d} t}\right|_{t=0} \exp t \xi \cdot m$ denotes the infinitesimal generator of the $\mathbb{T}^{n}$-action associated to the element $\xi$.

\section{Preliminaries}

Throughout we assume that $(\mathcal{M}, \omega)$ is a finite-dimensional symplectic manifold with a free Hamiltonian action of the torus $\mathbb{T}^{n}$ with momentum map $\mathbf{J}_{\mathbb{T}^{n}}: \mathcal{M} \rightarrow\left(\mathfrak{t}^{n}\right)^{*}$. Such a momentum map is necessarily invariant: $\mathbf{J}(g \cdot m)=\mathbf{J}(m)$ (with $g \in \mathbb{T}^{n}$ and $m \in \mathcal{M}$ ). We fix a torus subgroup $\mathbb{T}^{r} \subset \mathbb{T}^{n}$, and let $i: \mathfrak{t}^{r} \hookrightarrow \mathfrak{t}^{n}$ be the inclusion of Lie algebras. The momentum map for the restricted action by $\mathbb{T}^{r}$ is $\mathbf{J}_{\mathbb{T}^{r}}=i^{*} \circ \mathbf{J}_{\mathbb{T}^{n}}$, where $i^{*}:\left(\mathfrak{t}^{n}\right)^{*} \rightarrow\left(\mathfrak{t}^{r}\right)^{*}$ is the dual map to $i$.

We also assume that $m \in \mathcal{M}$ is a relative equilibrium for the Hamiltonian system $\left(\mathcal{M}, \omega, H_{0}, \mathbb{T}^{n}, \mathbf{J}_{\mathbb{T}^{n}}\right)$, with momentum $\mu:=\mathbf{J}_{\mathbb{T}^{n}}(m) \in\left(\mathfrak{t}^{n}\right)^{*}$ and velocity $\xi \in \mathfrak{t}^{n}$. We recall that this amounts to the point $m$ being a critical point of the augmented Hamiltonian $H_{0}-\mathbf{J}_{\mathbb{T}^{n}}^{\xi}$, that is, $D\left(H_{0}-\mathbf{J}_{\mathbb{T}^{n}}^{\xi}\right)(m)=0$.

In order to formulate the main hypothesis of the theorem we need to recall the Witt-Artin decomposition of the tangent space $T_{m} \mathcal{M}$; define $V_{m}$ as a complement in $\operatorname{ker} T_{m} \mathbf{J}_{\mathbb{T}^{n}}$ to the tangent space $\mathfrak{t}^{n} \cdot m$ at $m$ of the $\mathbb{T}^{n}$-group orbit, that is, $\operatorname{ker} T_{m} \mathbf{J}_{\mathbb{T}^{n}}=V_{m} \oplus \mathfrak{t}^{n} \cdot m$. The space $V_{m}$ is called the symplectic normal space at $m$. Notice that $\mathfrak{t}^{n} \cdot m \subset\left(V_{m}\right)^{\omega(m)}$. Let $W$ be a Lagrangian complement to $\mathfrak{t}^{n} \cdot m$ in $\left(V_{m}\right)^{\omega(m)}$. The decomposition

$$
T_{m} \mathcal{M}=V_{m} \oplus \mathfrak{t}^{n} \cdot m \oplus W
$$

is called a Witt-Artin decomposition of the tangent space $T_{m} \mathcal{M}$. We will refer to $W$ as the orbital complement at $m$ of the Witt-Artin decomposition (1). To finish these preliminaries, we give a definition which we will use in our result.

Definition 2.1. With the notation as above, a nondegeneracy space $\mathcal{N}_{\alpha}$ at $m$ associated to the momentum $\alpha \in\left(\mathfrak{t}^{r}\right)^{*}$ is defined as

$$
\mathcal{N}_{\alpha}=A_{\alpha} \oplus V_{m},
$$

where $A_{\alpha}:=\left\{w \in W \mid i^{*}\left(\mu+T_{m} \mathbf{J}_{\mathbb{T}^{n}}(w)\right)=\alpha\right\}$. Let $H \in C^{\infty}(\mathcal{M})^{\mathbb{T}^{n}}$ be a smooth $\mathbb{T}^{n}$-invariant function on $\mathcal{M}$ that exhibits a critical point at $m$, that is, $D H(m)=0$. We say that $m$ is an $\alpha$-nondegenerate critical point of $H$ when the symmetric bilinear form

$$
\left.D^{2} H(m)\right|_{\mathcal{N}_{\alpha} \times \mathcal{N}_{\alpha}}
$$

is nondegenerate.

Lemma 2.2. The $\alpha$-nondegeneracy of a critical point given in the previous definition depends only on the value $\alpha \in\left(\mathfrak{t}^{r}\right)^{*}$ and not on the specific Witt-Artin decomposition used to verify this condition.

Proof. It suffices to show that our nondegeneracy condition is independent of the choice of $V_{m}$ and $W$ in the Witt-Artin decomposition. Assume that $H$ is $\alpha$-nondegenerate at $m$ for a fixed choice of $V_{m}$ and $W$. Let $V_{m}^{\prime}$ be 
another choice of symplectic normal space at $m, W^{\prime}$ a complement to $\mathfrak{t}^{n} \cdot m$ in $\left(V_{m}^{\prime}\right)^{\omega(m)}$, and $\mathcal{N}_{\alpha}^{\prime}$ the associated nondegeneracy space. Let $v_{1}+w_{1}, v_{2}+w_{2} \in \mathcal{N}_{\alpha}$ be arbitrary with $v_{1}, v_{2} \in A_{\alpha}$ and $w_{1}, w_{2} \in V_{m}$. The Witt-Artin decomposition of $T_{m} \mathcal{M}$ implies the existence of unique elements $\xi, \eta \in \mathfrak{t}^{n}, v_{1}^{\prime}, v_{2}^{\prime} \in A_{\alpha}^{\prime}, w_{1}^{\prime}, w_{2}^{\prime} \in V_{m}^{\prime}$ such that

$$
v_{1}+w_{1}=\xi_{\mathcal{M}}(m)+v_{1}^{\prime}+w_{1}^{\prime} \quad \text { and } \quad v_{2}+w_{2}=\eta_{\mathcal{M}}(m)+v_{2}^{\prime}+w_{2}^{\prime}
$$

The $\mathbb{T}^{n}$-invariance of $H$ implies that

$$
D^{2} H(m)\left(v_{1}+w_{1}, v_{2}+w_{2}\right)=D^{2} H(m)\left(v_{1}^{\prime}+w_{1}^{\prime}, v_{2}^{\prime}+w_{2}^{\prime}\right) .
$$

Given that the map $v+w \in \mathcal{N}_{\alpha} \mapsto v^{\prime}+w^{\prime} \in \mathcal{N}_{\alpha}^{\prime}$ is an isomorphism, the result follows.

\section{Theorem on forced symmetry breaking}

The goal of this section is to prove the following theorem:

Theorem 3.1. Let $\left(\mathcal{M}, \omega, \mathbb{T}^{n}, \mathbf{J}_{\mathbb{T}^{n}}, \mathbb{T}^{r}, H_{0}, m, \xi, \mu\right)$ be as above. Let $H_{\varepsilon}$ be a family of $\mathbb{T}^{r}$-invariant Hamiltonian functions on $\mathcal{M}$ smoothly parametrized by $\varepsilon \in \mathbb{R}$ with $H_{0} \mathbb{T}^{n}$-invariant. Suppose that the relative equilibrium $m \in \mathcal{M}$ has velocity $\xi$. If

(i) $\xi \in i\left(\mathfrak{t}^{r}\right)$ and

(ii) $m$ is an $i^{*} \mu$-nondegenerate critical point of $H_{0}-\mathbf{J}_{\mathbb{T}^{n}}^{\xi}$, where $\mu:=\mathbf{J}_{\mathbb{T}^{n}}(m)$,

then for any value of the parameter $\varepsilon$ close enough to zero, the relative equilibria of the Hamiltonian vector field $X_{H_{\varepsilon}}$ are in bijective correspondence with the critical points of a smooth function $\bar{h}_{\varepsilon}: \mathbb{T}^{n-r} \rightarrow \mathbb{R}$. Consequently, under these hypotheses there exist at least $n-r+1$ relative equilibria of $X_{H_{\varepsilon}}$ with momentum $i^{*} \mu$ and velocity close to $\xi$. Additionally, if the critical points of $\bar{h}_{\varepsilon} \in C^{\infty}\left(\mathbb{T}^{n-r}\right)$ are all nondegenerate the number of bifurcating relative equilibria is at least $2^{\text {n-r }}$.

Proof. The local character of the result that we want to prove permits us to use the local model around the $\mathbb{T}^{n}$-orbit of $m$ given by the Marle-Guillemin-Sternberg (MGS) normal form [1,3]. We recall that this result provides a $\mathbb{T}^{n}$-equivariant symplectomorphism between a $\mathbb{T}^{n}$-invariant neighborhood of the orbit $\mathbb{T}^{n} \cdot m$ and the product $Y:=\mathbb{T}^{n} \times\left(\mathfrak{t}^{n}\right)^{*} \times V_{m}$, considered as a $\mathbb{T}^{n}$-symplectic space with the $\mathbb{T}^{n}$-action given by $g \cdot(h, \eta, v):=$ $(g h, \eta, v), g, h \in \mathbb{T}^{n}, \eta \in\left(\mathfrak{t}^{n}\right)^{*}, v \in V_{m}$ and with a symplectic form with respect to which the momentum map associated to this $\mathbb{T}^{n}$-action has the form $\mathbf{J}_{\mathbb{T}^{n}}(g, \eta, v)=\mu+\eta$. In this model, the point $m \in \mathcal{M}$ is represented by $(e, 0,0) \in Y$ and the space $V_{m}$ is one of the symplectic normal spaces at $m$ that we have previously defined. We will carry out the proof of our theorem in these coordinates by looking for the critical points of the reduced Hamiltonians $h_{\varepsilon, \alpha}$ on the $\mathbb{T}^{r}$-Marsden-Weinstein reduced space $\mathcal{M}_{\alpha}:=\mathbf{J}_{\mathbb{T}^{r}}^{-1}(\alpha) / \mathbb{T}^{r}$ defined by $h_{\varepsilon, \alpha} \circ \pi_{\alpha}=H_{\varepsilon} \circ i_{\alpha}$, where $\alpha:=i^{*} \mu, i_{\alpha}: \mathbf{J}_{\mathbb{T}^{r}}^{-1}(\alpha) \hookrightarrow \mathcal{M}$ is the injection, and $\pi_{\alpha}: \mathbf{J}_{\mathbb{T}^{r}}^{-1}(\alpha) \rightarrow \mathcal{M}_{\alpha}$ is the projection. A straightforward computation in MGS coordinates shows that

$$
\mathbf{J}_{\mathbb{T}^{r}}^{-1}(\alpha)=\mathbb{T}^{n} \times A_{\alpha} \times V_{m}
$$

where $A_{\alpha}$ is the vector subspace of $\left(\mathfrak{t}^{n}\right)^{*}$ given by $A_{\alpha}:=\left\{\eta \in\left(\mathfrak{t}^{n}\right)^{*} \mid i^{*}(\mu+\eta)=\alpha\right\}=\operatorname{ker} i^{*}$ and that $\mathcal{N}_{\alpha}:=A_{\alpha} \times V_{m}$ is a $\alpha$-nondegeneracy space at $m$. From expression (2) it is clear that

$$
\mathcal{M}_{\alpha}=\mathbb{T}^{n} \times A_{\alpha} \times V_{m} / \mathbb{T}^{r} \simeq \mathbb{T}^{n-r} \times \mathcal{N}_{\alpha}
$$

The problem of finding the relative equilibria in the statement of the theorem is now equivalent to the search for the critical points of the real-valued functions $h_{\varepsilon, \alpha}$ defined on the Marsden-Weinstein reduced space $\mathcal{M}_{\alpha}=$ $\mathbb{T}^{n-r} \times \mathcal{N}_{\alpha}$. 
The hypothesis on the $\alpha$-nondegeneracy of $m$ as a critical point of $H_{0}-\mathbf{J}_{\mathbb{T}^{n}}^{\xi}$ implies that the quadratic form $\left.D^{2} h_{0, \alpha}(e, 0,0)\right|_{\mathcal{N}_{\alpha} \times \mathcal{N}_{\alpha}}$ is nondegenerate. In order to lighten the notation we will omit the symbol $\alpha$ in the function $h_{\varepsilon, \alpha}$ in all that follows. With this notation, we need to find the triples $(k, \tilde{v}, \varepsilon) \in \mathbb{T}^{n-r} \times \mathcal{N}_{\alpha} \times \mathbb{R}$ such that

$$
D h_{\varepsilon}(k, \tilde{v})=0 .
$$

We proceed by using the Implicit Function Theorem to eliminate the parameter $\tilde{v} \in \mathcal{N}_{\alpha}$ from Eq. (3) by writing it in terms of the $\mathbb{T}^{n-r}$ and $\mathbb{R}$ variables. Indeed, consider the following map

$$
\begin{aligned}
\mathcal{F}: \mathbb{T}^{n-r} \times \mathcal{N}_{\alpha} \times \mathbb{R} & \rightarrow\left(\mathcal{N}_{\alpha}\right)^{*} \simeq \mathcal{N}_{\alpha}, \\
(k, \tilde{v}, \varepsilon) & \mapsto D_{\mathcal{N}_{\alpha}} h_{\varepsilon}(k, \tilde{v}) .
\end{aligned}
$$

Since $m \equiv(e, 0,0)$ is a $\mathbb{T}^{n}$-relative equilibrium for $H_{0}$ we have $\mathcal{F}(g, 0,0)=0$, for all $g \in \mathbb{T}^{n-r}$. Moreover, since the partial derivative $D_{\mathcal{N}_{\alpha}} \mathcal{F}(g, 0,0): \mathcal{N}_{\alpha} \rightarrow\left(\mathcal{N}_{\alpha}\right)^{*} \simeq \mathcal{N}_{\alpha}$ of $\mathcal{F}$ with respect to the $\mathcal{N}_{\alpha}$-factor, evaluated at $(g, 0,0)$ is given by $D_{\mathcal{N}_{\alpha}} \mathcal{F}(g, 0,0)=D_{\mathcal{N}_{\alpha}}^{2} h_{0}(g, 0)$ then the hypothesis on the $\alpha$-nondegeneracy of $m$ as a critical point of $H_{0}-\mathbf{J}_{\mathbb{T}^{n}}^{\xi}$ implies that $D_{\mathcal{N}_{\alpha}} \mathcal{F}(g, 0,0): \mathcal{N}_{\alpha} \rightarrow \mathcal{N}_{\alpha}$ is injective. Consequently, $D_{\mathcal{N}_{\alpha}} \mathcal{F}(g, 0,0)$ is an isomorphism and we can then define via the Implicit Function Theorem a smooth map $\tilde{v}_{g}: \mathcal{U}_{g} \times \mathcal{W}_{g} \rightarrow\left(\mathcal{N}_{\alpha}\right)_{g} \subset \mathcal{N}_{\alpha}$ defined in an open neighborhood of $(g, 0) \in \mathbb{T}^{n-r} \times \mathbb{R}$ such that, for any $(k, \varepsilon) \in \mathbb{T}^{n-r} \times \mathbb{R}$ in that neighborhood, we have that:

$$
D_{\mathcal{N}_{\alpha}} h_{\varepsilon}\left(k, \tilde{v}_{g}(k, \varepsilon)\right)=D_{\mathcal{N}_{\alpha}} h_{\varepsilon}\left(k, \tilde{v}_{g, \varepsilon}(k)\right)=0 .
$$

Given that this argument can be repeated for any $g \in \mathbb{T}^{n-r}$ we can invoke the compactness of $\mathbb{T}^{n-r}$ to build a finite family of functions $\tilde{v}_{g_{i}}: \mathcal{U}_{g_{i}} \times \mathcal{W}_{g_{i}} \rightarrow\left(\mathcal{N}_{\alpha}\right)_{g_{i}}, i \in\{1, \ldots, \ell\}$, satisfying (4) and such that $\bigcup_{i=1}^{\ell} \mathcal{U}_{g_{i}}=\mathbb{T}^{n-r}$. Let us define

$$
\begin{aligned}
\tilde{v}: \mathbb{T}^{n-r} \times \bigcap_{i=1}^{\ell} \mathcal{W}_{g_{i}} & \rightarrow \bigcup_{i=1}^{\ell}\left(\mathcal{N}_{\alpha}\right)_{g_{i}}, \\
(g, \varepsilon) & \mapsto \tilde{v}_{g_{i}}(g, \varepsilon) \text { if } g \in \mathcal{U}_{g_{i}} .
\end{aligned}
$$

This map is well defined by the uniqueness of the maps $\tilde{v}_{g_{i}}$ obtained from the Implicit Function Theorem. Taking into account this new map, our bifurcation equation (3) is now equivalent to:

$$
D h_{\varepsilon}(k, \tilde{v}(k, \varepsilon))=0 .
$$

The solutions of this equation coincide with the critical points of the function $\bar{h}_{\varepsilon}(k):=h_{\varepsilon}(k, \tilde{v}(k, \varepsilon))$ defined, for each value of the parameter $\varepsilon$, on the compact manifold $\mathbb{T}^{n-r}$. Indeed, using (4), we have

$$
\begin{aligned}
D \bar{h}_{\varepsilon}(t) & =D_{\mathbb{T}^{n-r}} h_{\varepsilon}(t, \tilde{v}(t, \varepsilon))+D_{\mathcal{N}_{\alpha}} h_{\varepsilon}(t, \tilde{v}(t, \varepsilon)) \cdot D_{\mathbb{T}^{n-r}} \tilde{v}(t, \varepsilon)=D_{\mathbb{T}^{n-r}} h_{\varepsilon}(t, \tilde{v}(t, \varepsilon)) \\
& =D_{\mathbb{T}^{n-r}} h_{\varepsilon}(t, \tilde{v}(t, \varepsilon))+D_{\mathcal{N}_{\alpha}} h_{\varepsilon}(t, \tilde{v}(t, \varepsilon))=D h_{\varepsilon}(t, \tilde{v}(t, \varepsilon)) .
\end{aligned}
$$

Consequently, the pair $(t, \tilde{v}(t, \varepsilon))$ is a solution of (5) if and only if $t \in \mathbb{T}^{n-r}$ is a critical point of $\bar{h}_{\varepsilon}$. A lower bound for the number of these critical points is provided by the Ljusternik-Schnirelmann category Cat $\left(\mathbb{T}^{n-r}\right)=n-r+1$ of the torus $\mathbb{T}^{n-r}$ (see, for instance, [2]), which proves the statement of the theorem. Additionally, if we know in advance that the critical points of $\bar{h}_{\varepsilon} \in C^{\infty}\left(\mathbb{T}^{n-r}\right)$ are all nondegenerate, the Morse inequalities guarantee that this function has at least $b^{0}\left(\mathbb{T}^{n-r}\right)+b^{1}\left(\mathbb{T}^{n-r}\right)+\cdots+b^{n-r}\left(\mathbb{T}^{n-r}\right)$ critical points, where $b^{i}\left(\mathbb{T}^{n-r}\right), i \in\{0, \ldots, n-r\}$, is the $i$-th Betti number of the torus $\mathbb{T}^{n-r}$. Since $b^{i}\left(\mathbb{T}^{n-r}\right)=\left(\begin{array}{c}n-r \\ i\end{array}\right), i \in\{0, \ldots, n-r\}$, we have

$$
b^{0}\left(\mathbb{T}^{n-r}\right)+b^{1}\left(\mathbb{T}^{n-r}\right)+\cdots+b^{n-r}\left(\mathbb{T}^{n-r}\right)=\sum_{i=0}^{n-r}\left(\begin{array}{c}
n-r \\
i
\end{array}\right)=2^{n-r}
$$

and hence the second estimate in the statement follows. 


\section{Symmetry breaking using Poisson reduction}

In the previous theorem we confined our search for bifurcated relative equilibria to the momentum level set $\mathbf{J}_{\mathbb{T}^{r}}^{-1}\left(i^{*} \mu\right)$. This fact appears in the proof of that result when we use the symplectic reduced space $\mathcal{M}_{i^{*} \mu}$. If instead of using $\mathcal{M}_{i^{*} \mu}$ we consider the Poisson reduced space $\widetilde{\mathcal{M}}:=\mathcal{M} / \mathbb{T}^{r}$ we can obtain another bifurcation result where the predicted relative equilibria could, in principle, have a momentum different from that of the given RE. This is obtained at the expense of imposing a more demanding nondegeneracy condition.

Theorem 4.1. Let $\left(\mathcal{M}, \omega, \mathbb{T}^{n}, \mathbf{J}_{\mathbb{T}^{n}}, \mathbb{T}^{r}, H_{0}, m, \xi, \mu\right)$ be as in Section 2. Let $H_{\varepsilon}$ be a family of Hamiltonian functions on $\mathcal{M}$ parametrized by $\varepsilon \in \mathbb{R}$ and assume that $H_{0}$ is $\mathbb{T}^{n}$-invariant whereas $H_{\varepsilon}$ is only $\mathbb{T}^{r}$-invariant, for all $\varepsilon \in \mathbb{R}$. Suppose that the point $m \in \mathcal{M}$ is a $\mathbb{T}^{n}$-relative equilibrium of the Hamiltonian vector field $X_{H_{0}}$ with velocity $\xi \in i\left(\mathfrak{t}^{r}\right)$. Suppose moreover that

$$
\left.D^{2}\left(H_{0}-\mathbf{J}_{\mathbb{T}^{n}}^{\xi}\right)(m)\right|_{\mathcal{N} \times \mathcal{N}}
$$

is a nondegenerate quadratic form, where $\mathcal{N}:=W \times V_{m}$, for the symplectic normal space $V_{m}$ and orbital complement $W$ corresponding to some Witt-Artin decomposition of $T_{m} \mathcal{M}$. Then for any value of the parameter $\varepsilon$ close enough to zero, the relative equilibria of the Hamiltonian vector field $X_{H_{\varepsilon}}$ are in bijective correspondence with the critical points of a smooth function $\left[h_{\varepsilon}\right]: \mathbb{T}^{n-r} \rightarrow \mathbb{R}$. Consequently, under these hypotheses there exist at least $n-r+1 \mathbb{T}^{r}$-relative equilibria near $m$ with momentum close to $i^{*} \mu$ and velocity close to $\xi$. Additionally, if the critical points of $\left[h_{\varepsilon}\right] \in C^{\infty}\left(\mathbb{T}^{n-r}\right)$ are all nondegenerate the number of these bifurcated relative equilibria is at least $2^{\text {n-r }}$.

Proof. This mimics the proof of Theorem 3.1 where the reduced space $\mathcal{M}_{\alpha}$ has been replaced by $\widetilde{\mathcal{M}}$. Note that in the MGS normal form coordinates we can write, locally,

$$
\widetilde{\mathcal{M}}=\left(\mathbb{T}^{n} \times\left(\mathfrak{t}^{n}\right)^{*} \times V_{m}\right) / \mathbb{T}^{r} \simeq \mathbb{T}^{n-r} \times\left(\mathfrak{t}^{n}\right)^{*} \times V_{m}
$$

\section{Acknowledgements}

This research was partially supported by the European Commission through funding for the Research Training Network Mechanics and Symmetry in Europe (MASIE).

\section{References}

[1] V. Guillemin, S. Sternberg, A normal form for the moment map, in: S. Sternberg (Ed.), Differential Geometric Methods in Mathematical Physics, in: Math. Phys. Stud., vol. 6, Reidel, 1984.

[2] L.A. Ljusternik, The Topology of the Calculus of Variations in the Large, in: Transl. Math. Monographs, vol. 16, American Mathematical Society, 1966.

[3] C.-M. Marle, Le voisinage d'une orbite d'une action hamiltonienne d'un groupe de Lie, in: P. Dazord, N. Desolneux-Moulis (Eds.), Séminaire Sud-Rhodanien de Géométrie II, 1984, pp. 19-35. 